\title{
Research on the Design and Implementation of Distributed Electrical Control Systems
}

\author{
ZHANG Yuchen ${ }^{1, a}$ \\ ${ }^{1}$ Shenyang Institute of Engineering, Shenyang 110121, China \\ azhangyuchen@qq.com
}

Keywords: control system, electronic system, interaction control.

\begin{abstract}
The consolidation of the applicability of the method of communication, has the ability to coordinate the internal mode conversion only rely on distributed PLS signal DEM system has been developed. It has been shown, this coordination can be used for a plurality of battery application and Study on method of scalability, and at the same time management more units are discussed from the angle of the control more senior and brought from the viewpoint of system dynamics limitation. Our research focuses on distributed electrical control systems and the experimental analysis shows the feasibility of our research.
\end{abstract}

\section{Introduction}

Present stage of information technology development is characterized by a sharp jump in the production of devices for thermal treatment of various kinds of products. Although in the recent past, characterized by low efficiency of heat treatment equipment, and the defects of the control system of temperature field, at present, due to the introduction of cylindrical dryer, cross-system interaction control system increases the room (for example, oven by driver group "Kantal") [1-5]. Temperature field control the tray temperature will still not solve the problem. The control problem of solution is not, however, may not solve the problem of heating and as a result of temperature field supervision aspect more bay. Complexity depends on the complexity of the implementation of the object of this study and the distributed control system parameters. The control quantity of an order for the system depends not only on time, but also take up space in the spatial distribution of the object. In this regard, basically control behaviour scope expanding, mainly due to the possibility of including the control of time and space, is the function of several variables, namely, time and space coordinates.

In general, dc systems offer several advantages over their ac counterparts, such as increased efficiency and the non-existence of synchronization and reactive power flow issues [6]. Today, power supply of remote sites like telecom stations and data centres, where reliability and power quality are of great importance, is almost exclusively achieved with utilization of dc distribution [7]. Particularly interesting to solve this kind of system is the concept of power use only magazine, however RES force and variable nature of the balance of power challenge of isolated action [9-12]. The addition of energy storage system (ESS), such as secondary (rechargeable) battery, is an option for maintaining the power balance continuously in small and autonomous systems. Regardless on internal technology, the price of the battery string generally plays an important part in the overall cost here and a special care should be therefore taken to preserve its lifetime. In this context, it is best practice to realize charging method is put forward from the battery manufacturers, avoid frequent deep discharge cycles. However, once the regulated charging process starts, battery loses its power balance with the current extraction from the internal control circuit is decided on the grid. If you enable the activation of the event monitoring, lack of enough ability to provide load or stable problem may occur in the weak system. In addition, if have more battery serial parallel connection, a coordinated strategy becomes mandatory. Not achieve the desired reactive power injection, apparent power inverter saturation capacity. In a centralized approach, the optimal recovery of the central authority, because the complete observability make it realize saturated allows it to generate additional reactive power compensation (or consumption) near nodes. Think there are other reasons 
limited communication can provide significant advantages over based on the strategy of control no concentration of large communication overhead. Mainly to adapt to the system configuration, and do not foresee the development of the policy is not based on heuristic or sampling method.

In order to tackle the issues of previously mentioned strategies, a method based on power-line signalling (PLS) is proposed in this paper. The concept of power-line communication (PLC) has been widely used by electric utility companies to shut off the corrupted parts of the network and for information transmission. We discuss the problems in detail in the next sections.

\section{Proposed Methodology}

Architecture and Control. Fig. 1 represents an autonomous LVDC MG formed around a common dc bus to which the sources and loads are directly connected. Typical load can be roughly divided into passive and active, but they are usually designed for a specific major bus voltage. Voltage - droop (VD) method is a widely accepted way to obtain parallel operation control using a variety of sources. Operation principle is the resources to control the public bus voltage according to the introduction of the total consumption of internal virtual resistance Rd as control parameters shown in the following figure 2.

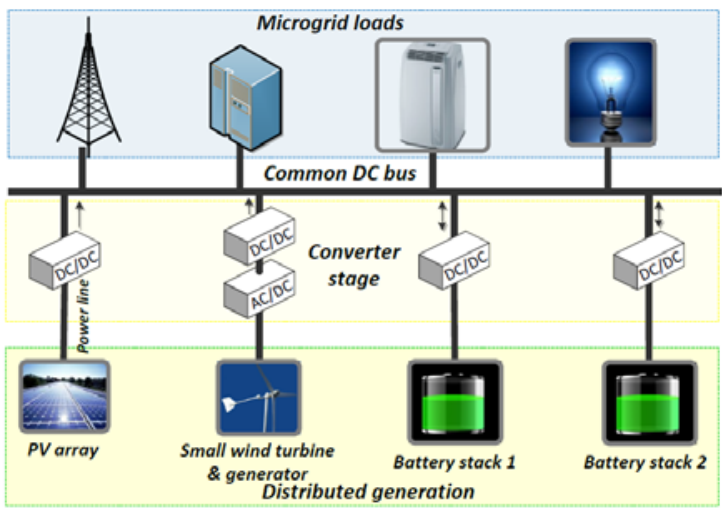

Figure 1: The Diagram of a Small-scale LVDC Micro-grid

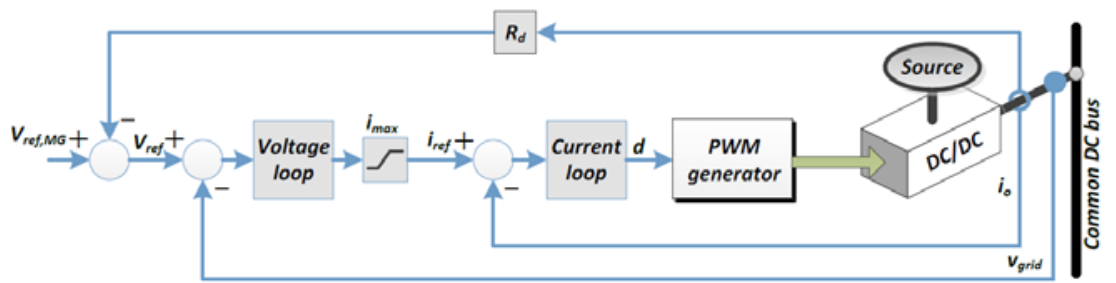

Figure 2: The Block Diagram of a VD Unit by:

In this way, it is ensured that the steady-state operating point of the unit stays on the line defined

$$
v_{D C}=v_{\text {ref }, M G}-R_{d} i_{o}
$$

However, depending on the role of each unit, VD operation may not always be the best control strategy. For instance, instead of using RES for voltage support, it is better to extract all available free power from them with the use of dedicated maximum power point tracking (MPPT) algorithms whenever is possible. Up to date, a number of control strategies for that purpose has been developed for both PV and WTG plants. Breaking the symmetry is relatively sensitive, small mistake can create a big obstacle, approximate to change the type of the PDE. Uncertainty, by contrast, remained relatively induction symmetry (such as the uncertainty in each sensor gain) is easy to prove that is less harmful to the robust stability. Many physical situations, however, seems can lead to uncertainty of the model, the violation. The main source voltage circuit which ensure the tracking their respective reference voltage and reference current internal control circuit. On the other hand, in order to restore the connection of the battery charge (SOC), constant-voltage charge process 
method should be used. More specifically, a two-stage constant pressure control type sealed lead acid battery charging method is the most suitable technology, is still one of the most common application of fixed. The first high voltage - stage setting, commonly referred to as "stimulus" voltage, and another is commonly referred to as "float" voltage. Suggest specific voltage values and their respective duration is usually provided by the specific battery manufacturers. In order to do this, the internal cell cycle control circuit including voltage and current are active in this process. Battery voltage circuit decision required reference current levels necessary to sustain the battery voltage.

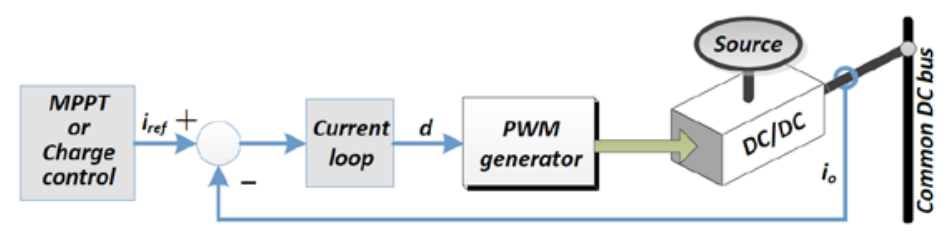

Figure 3: The Block Diagram of a CP Unit

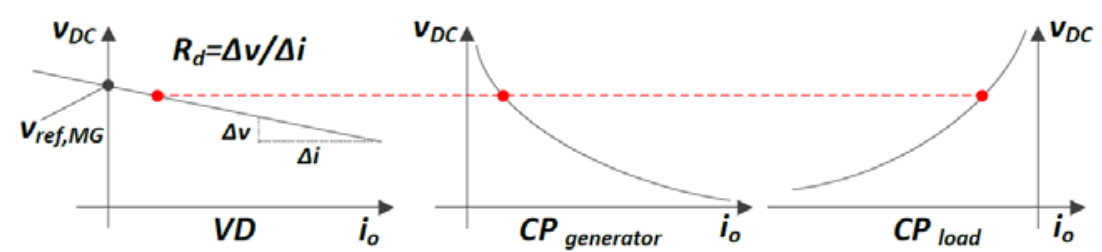

Figure 4: The Static Characteristics of a VD, CP Generator and CP

Load Units Connected to the Common dc Bus

Even though it can be seen from the Fig. 3 that associated unit is current controlled, the process of calculation of its input current reference makes it virtually a constant power (CP) unit in the static sense. So, the MPPT algorithm will continuously adjust the RES voltage reference so as to keep extracting maximum power from it. On the other hand, charge algorithm will maintain the regulated charging of the battery. Both of these powers may be considered as constant for given environmental conditions and status of the battery. The static characteristics of all types of aforementioned units in i-v plane are shown in the previous figure 4.

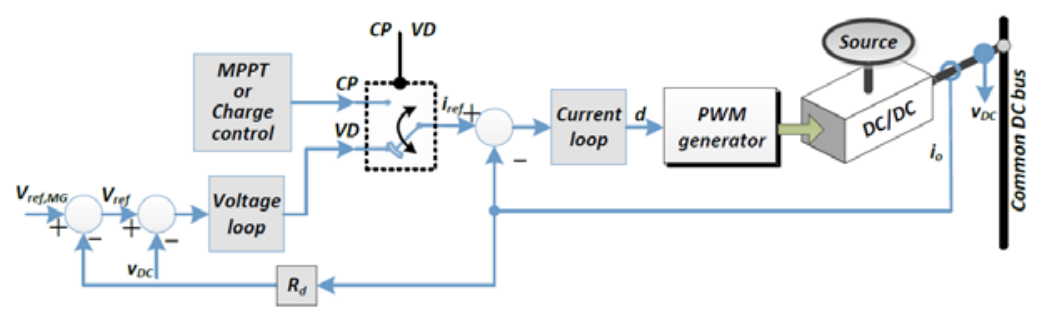

Figure 5: The Block diagram of a source

Controls of connected units have been designed so that the sources of reference currents can be dynamically overlapped externally, as shown in Fig. 5. Thus, depending on external signal, every source may operate in VD or CP mode, with the currently active mode being termed as the unitlevel (UL) mode. It should be noted that RES output power in VD mode should not exceed its maximum available power and that compliance is achieved by providing dynamic limiters to current control loops. Moreover, smooth transitions between UL modes in both ways were ensured by automatic setting of appropriate initial conditions for associated controller integrators. In next section, a communication concept based on exchange of PLS messages between the units is developed.

The Applicability. Bode diagrams can provide help in the selection of optimum frequency values. In order to construct them, a full set of parameters for every converter needs to be specified. In this particular study, the selection of LC filters was governed by the switching frequency of available real-time control platform, which is $10 \mathrm{kHz}$. Fig. 6 . One may observe from the figure the magnitude peaks occurring at frequencies between 150 and $220 \mathrm{~Hz}$. They denote the frequencies for which the mapping of the input reference to output voltage is achieved with minimum additional 
current loading to converters. According to the response, also the frequencies between 100 and 250 $\mathrm{Hz}$ can be considered to be in the favorable frequency region. Therefore, it is advisable to choose the frequencies in that range. However, it is worth mentioning that different switching frequencies of particular converters would imply selection of distinctive filters and hence the settings of control parameters.

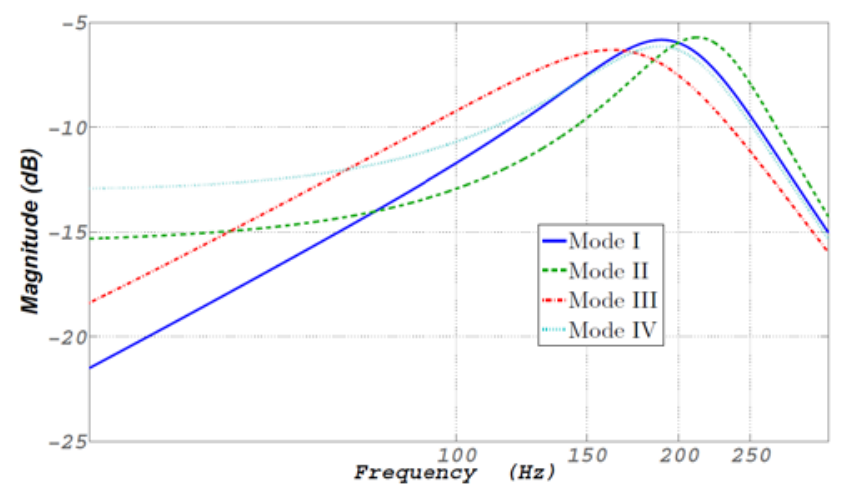

Figure 6: The Frequency Magnitude Responses

The selected frequencies should not be to close to each other so as to have a clear differentiation of the PLS signals. Also, the minimum PLS frequency should not be too low in order to avoid interaction with converters' primary control loops and not too close to $100 \mathrm{~Hz}$ as it could interact with optional AC loads. Having the aforementioned facts in mind, the main and auxiliary frequencies for battery 1 have been chosen as $113 \mathrm{~Hz}$ and $131 \mathrm{~Hz}$, and for battery 2 as $122 \mathrm{~Hz}$ and $140 \mathrm{~Hz}$. This way, still there is room to expand the concept with additional frequencies. However, if the frequency resources are exhausted, one may go out of the proposed range but taking care not to overburden the PLS signal injecting converters. The figure 7-8 shows this.

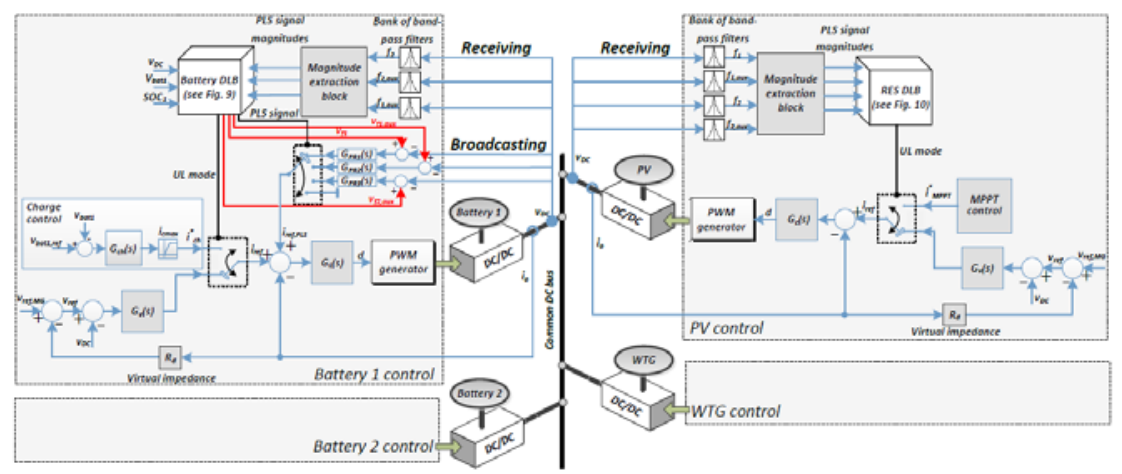

Figure 7: The Block diagram of the PLS

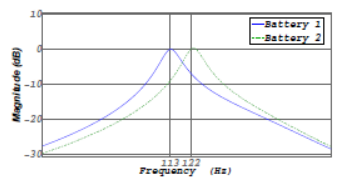

(a) Mode 1 - magnitude responses for frequencies $113 \mathrm{~Hz}$ and $122 \mathrm{~Hz}$

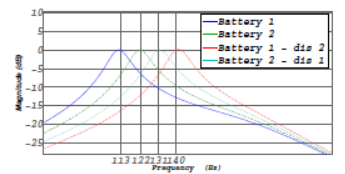

Figure 8: The Frequency Magnitude Responses

\section{The Experimental Analysis}

A dc MG prototype of Fig. 1 has been implemented and tested in the lab. Hardware platform was assembled from a dc power supply that fed four dc/dc synchronous buck converters, where every converter contains LC filter on the output, and all of them were connected in parallel to form a common dc bus. Electronic load was used to emulate the power deficiency periods. Control 
diagrams that correspond to elaboration in previous sections were developed in Matlab/Simulink and Matlab/Stateflow and were compiled to the dSPACE 1103, which was utilized for real-time control of converter switches. For the reason that the experiments with real PV array, wind turbine and batteries would be impractical due to long waiting times for batteries to charge and discharge and difficulties in invoking different scenarios for mode transitions with unpredictable RES production, they have been emulated in real time using dSPACE 1103 as well. The following figures shows this.
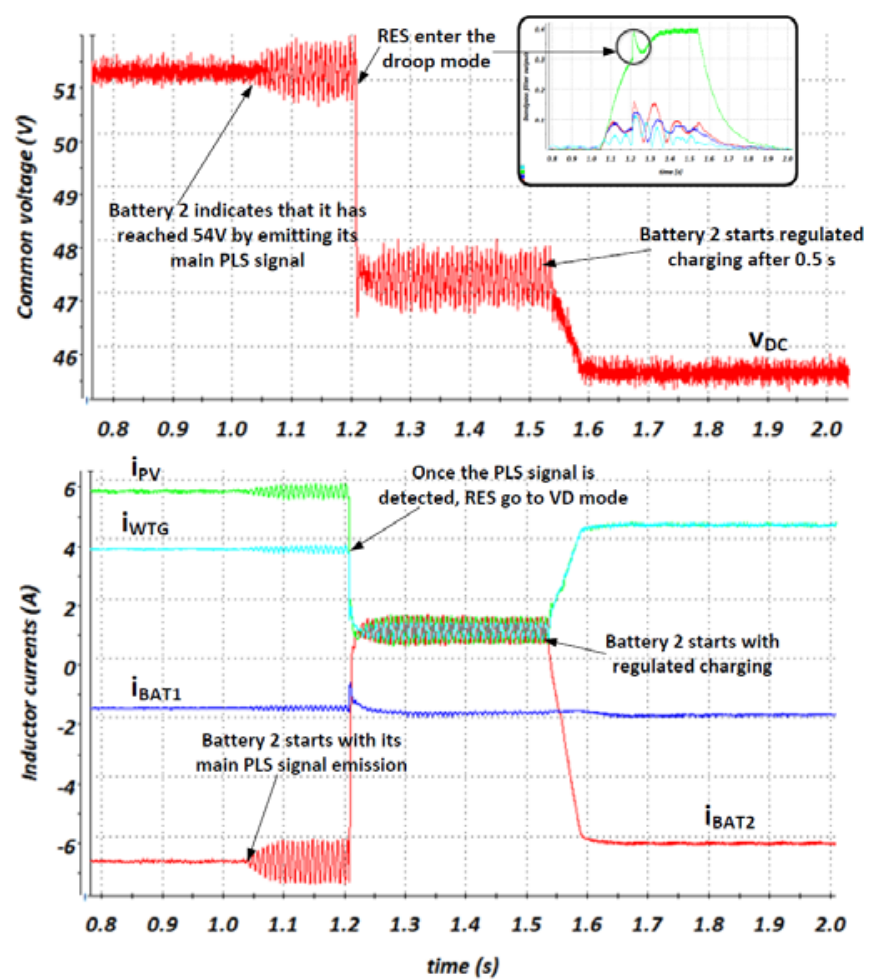

Figure 9: The Experimental Result

\section{Conclusions}

A PLS based DEMS for autonomous LVDC MGs has been proposed in this paper. Here, the power network serves as a communication channel for exchanging messages in the form of PLS signals which are injected directly from converters' primary control loops. The experimental result demonstrates the effectiveness of our proposed methodology and we will research this topic in detail in the near future.

\section{References}

[1] Lew, Roger, Ronald L. Boring, and Thomas A. Ulrich. "A prototyping environment for research on human-machine interfaces in process control use of Microsoft WPF for microworld and distributed control system development." Resilient Control Systems (ISRCS), 2014 7th International Symposium on. IEEE, 2014.

[2] Motus, L., and K. Kääramees. "A model based design of distributed control system software." Distributed Computer Control Systems 1982: Proceedings of the Fourth IFAC Workshop, Tallinn, USSR, 24-26 May 1982. Elsevier, 2014.

[3] Coron, Jean-Michel, and Pierre Lissy. "Local null controllability of the three-dimensional Navier-Stokes system with a distributed control having two vanishing components." Inventiones mathematicae 198.3 (2014): 833-880.

[4] Charles, C. Arnold, et al. "Study of 3MeV DC Electron Beam Accelerator to implement Distributed Control System." Journal for Foundations and Applications of Physics 1.1 (2014): 16-19. 
[5] Prabhavathy, M., B. Ramesh, and T. Kalpalatha Reddy. "An alternative distributed control using Labview." Computation of Power, Energy, Information and Communication (ICCPEIC), 2014 International Conference on. IEEE, 2014.

[6] Jian-Fu, Cao, et al. "Distributed formation control for a multi-agent system with dynamic and static obstacle avoidances." Chinese Physics B 23.7 (2014): 070509.

[7] Shafiee, Qobad, Josep M. Guerrero, and Juan C. Vasquez. "Distributed secondary control for islanded microgrids-A novel approach." Power Electronics, IEEE Transactions on 29.2 (2014): 1018-1031.

[8] Zhang, Wei, et al. "Design of Distributed Control System of Liquid Fermentation Based on S7300 PLC and WinCC." Applied Mechanics and Materials. Vol. 651. 2014.

[9] Dörfler, Florian, John Simpson-Porco, and Francesco Bullo. "Breaking the hierarchy: Distributed control \& economic optimality in microgrids." arXiv preprint arXiv:1401.1767 (2014).

[10] Carandina, Alessandro, et al. "A Distributed Control System for a Field of Spin-Elevation Heliostats." Advances in Condition Monitoring of Machinery in Non-Stationary Operations. Springer Berlin Heidelberg, 2014. 517-531.

[11] Muller, K. D., and M. G. Rodd, eds. Distributed Computer Control Systems 1986: Proceedings of the Seventh IFAC Workshop, Mayschoss/Bad Neuenahr, FRG, 30 September-2 October 1986. Elsevier, 2014.

[12] Welser, Howard T. "Breaking the Iron Law of Oligarchy: Computational Institutions, Organizational Fidelity, and Distributed Social Control." Roles, Trust, and Reputation in Social Media Knowledge Markets. Springer International Publishing, 2015. 121-144. 